\title{
Esophageal cancer diagnosed by high-resolution manometry of the esophagus: A case report
}

\author{
RONGBEI LIU ${ }^{1,2^{*}}$, HUA CHU $^{1 *}$, FEI XU ${ }^{1}$ and SHUJIE CHEN ${ }^{1,2}$ \\ ${ }^{1}$ Department of Gastroenterology, Sir Run Run Shaw Hospital, School of Medicine; \\ ${ }^{2}$ Institute of Gastroenterology, Zhejiang University, Hangzhou, Zhejiang 310000, P.R. China
}

Received January 20, 2015; Accepted February 24, 2016

DOI: $10.3892 / 01.2016 .4332$

\begin{abstract}
A 48-year-old female who presented with a history of dysphagia for 5 months and regurgitation for 1 week was referred to the Sir Run Run Shaw Hospital (Hangzhou, China) for further evaluation, since the gastroscopy and endoscopic ultrasound performed in local hospitals did not reveal the presence of cancer. High-resolution manometry (HRM) of the esophagus was performed to determine the patient's condition, and revealed an abnormal high-pressure zone that was located $33 \mathrm{~cm}$ from the incisor and did not relax upon swallowing. Synchronous waves were observed, and the pressure of the esophageal lumen was found to increase with secondary synchronous peristaltic waves. The lower esophageal sphincter was $39 \mathrm{~cm}$ from the incisor and relaxed upon swallowing. The abnormal high-pressure zone could have been caused by an obstruction, and therefore an upper gastrointestinal series (barium swallow) test and gastroscopy were recommended to further pinpoint the cause. Following the two examinations, mid-esophageal cancer was considered as a possible diagnosis. A biopsy was performed and the final diagnosis was that of basaloid squamous cell carcinoma. The findings of the present study suggest that, for patients with evident symptoms of esophageal motor dysfunction without significant gastroscopy findings, HRM is recommended.
\end{abstract}

\section{Introduction}

Esophageal cancer is the eighth most common type of cancer worldwide, and the incidence rate is 11.5 in 100,000 individuals for men and 4.7 in 100,000 individuals for women (1).

Correspondence to: Dr Shujie Chen, Department of Gastroenterology, Sir Run Run Shaw Hospital, School of Medicine, Zhejiang University, 3 East Qingchun Road, Hangzhou, Zhejiang 310000, P.R. China

E-mail: chensj77@hotmail.com

${ }^{*}$ Contributed equally

Key words: basaloid squamous cell carcinoma of the esophagus, esophageal neoplasms, high-resolution manometry of the esophagus
Histologically, esophageal cancers are divided into two primary types: Esophageal adenocarcinomas and esophageal squamous cell carcinomas, which are associated with the lower esophagus and between the middle and upper esophagus, respectively (2). The most common presenting symptom for esophageal cancer is progressive dysphagia for solids, which progresses to dysphagia for liquids over the course of weeks to months (3). Esophageal manometry is a common procedure used for the detection of esophageal motility disorders, and conventional pull-through and high-resolution manometry (HRM) are each used clinically. HRM is an advanced form of manometry that has been used in research and clinical practice (4). HRM is a device for esophageal pressure recording, and a common procedure used for the diagnosis of esophageal motility disorders, including achalasia, gastroesophageal reflux disease, esophageal hiatus hernia and nutcracker esophagus (5-7). HRM has multiple advantages, including ease of use, high sensitivity and accuracy in the analysis of detailed esophageal pressure topography $(8,9)$ compared with conventional pull-through manometry. The combination of HRM with other examinations, including gastroscopy, endoscopic ultrasound, barium esophagogram and impedance testing, may therefore assist the diagnosis of esophageal cancer.

The current study presents the case of a patient that had undergone an endoscopy and endoscopic ultrasound for dysphagia; however, no evidence of cancer was observed. Subsequently, an abnormal high-pressure zone was identified using HRM, leading to an endoscopy and biopsy of a tumor. Therefore, although HRM is usually used to diagnose esophageal motility disorders, in the present case it was used as an aid to diagnose esophageal cancer.

\section{Case report}

A 48-year-old female presented to the Sir Run Run Shaw Hospital (Hangzhou, China) in September 2011 with a history of dysphagia for 5 months and regurgitation for 1 week. At 5 months prior to this presentation, the patient had developed dysphagia with no clear cause, and no other symptoms were recorded. The patient did not have a history or smoking or heavy drinking. The patient visited a local hospital and underwent an upper endoscopy that revealed coarse mucosa in the region of the distal esophagus above the pectinate line, and dotted damaged mucosa distributed sporadically without 


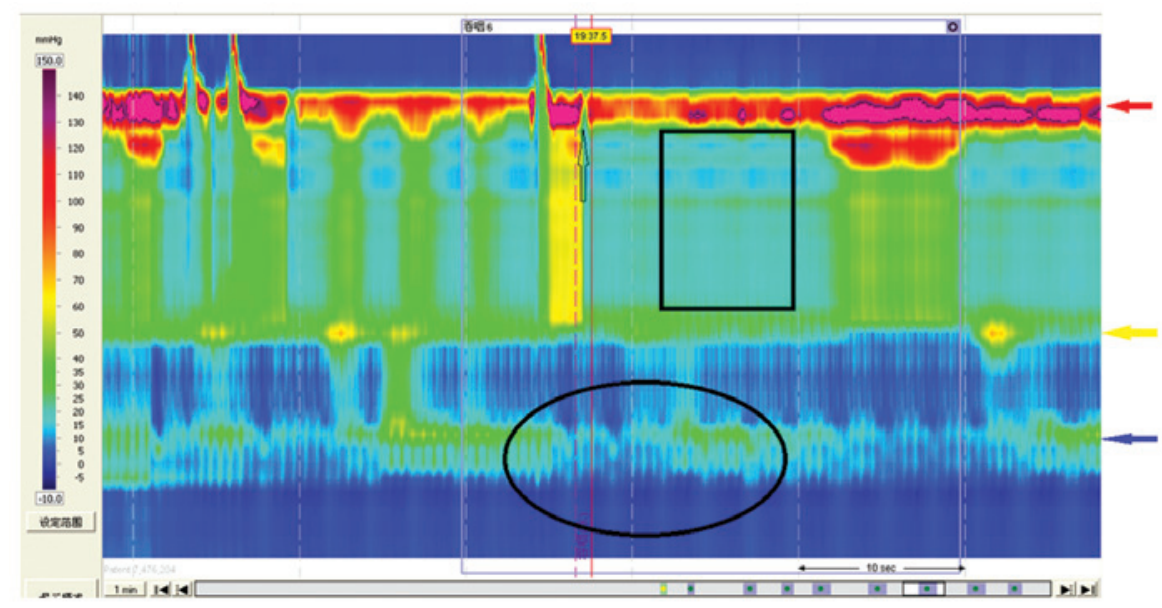

Figure 1. High-resolution esophageal manometry recording showing an abnormal increase in the upper esophageal sphincter pressure (red arrow) and an abnormal high-pressure zone $33 \mathrm{~cm}$ from the incisor (yellow arrow), above the high-pressure zone. Synchronous waves were observed upon swallowing (black arrows), together with secondary peristaltic waves. The peristaltic waves were shown to remain even without swallowing, and pressure in the esophageal lumen was increased (rectangle), causing food to remain. Partial liquid flow from the esophagus into the stomach through the high-pressure zone was noted, and the lower esophageal sphincter was shown to relax upon swallowing (circle).

diffusion. The lesion with the largest diameter measured $\sim 0.3 \mathrm{~cm}$. The patient was diagnosed with reflux esophagitis (grade A), antral polyps and chronic superficial gastritis with partial atrophy. Treatment with a combination of traditional Chinese (unknown) and Western medicines (40 mg pantoprazole once a day and $5 \mathrm{mg}$ mosapride three times a day for $\sim 15$ days) was applied, but no improvement was shown. At 3 months prior to presentation, the patient visited another local hospital and an endoscopic ultrasound examination revealed a lesion, $\sim 0.5-\mathrm{cm}$ in diameter, protruding into the anterior of the gastric antrum and mucosa, with a smooth surface and a small amount of surrounding swelling. A diagnosis of lesions protruding into the gastric antrum and a thickened muscular layer was considered. The patient continued to experience dysphagia and also presented with regurgitation 1 week prior to being admitted to the Sir Run Run Shaw Hospital for further evaluation. At the time of admission, weight loss of $5 \mathrm{~kg}$ over the last week was noted.

Upon physical examination, the lymph nodes in the bilateral supraclavicular region were palpable and measured $\sim 0.5 \mathrm{~cm}$ in diameter. Carbohydrate antigen 242 levels were found to be slightly elevated at $16.48 \mathrm{U} / \mathrm{ml}$ (normal values, $0.00-10.00 \mathrm{U} / \mathrm{ml}$ ), but all other blood chemistry results were normal. The HRM of the esophagus performed to clarify the patient's condition revealed an abnormal high-pressure zone that was located $33 \mathrm{~cm}$ from the incisor and did not relax upon swallowing (A100 Manometry Equipment; Sierra Scientific Instruments LLC, Los Angeles, CA, USA). Synchronous waves were observed, and the pressure of the esophageal lumen was found to increase with secondary synchronous peristaltic waves. The lower esophageal sphincter (LES) was $39 \mathrm{~cm}$ from the incisor and relaxed upon swallowing (Fig. 1). The abnormal high-pressure zone could have been caused by an obstruction, and therefore an upper gastrointestinal series (barium swallow) test and gastroscopy were recommended to further pinpoint the cause. The upper gastrointestinal series (barium swallow) test (digital radiography; AXIOM Aristos FX; Siemens Healthcare, Erlangen, Germany.) showed

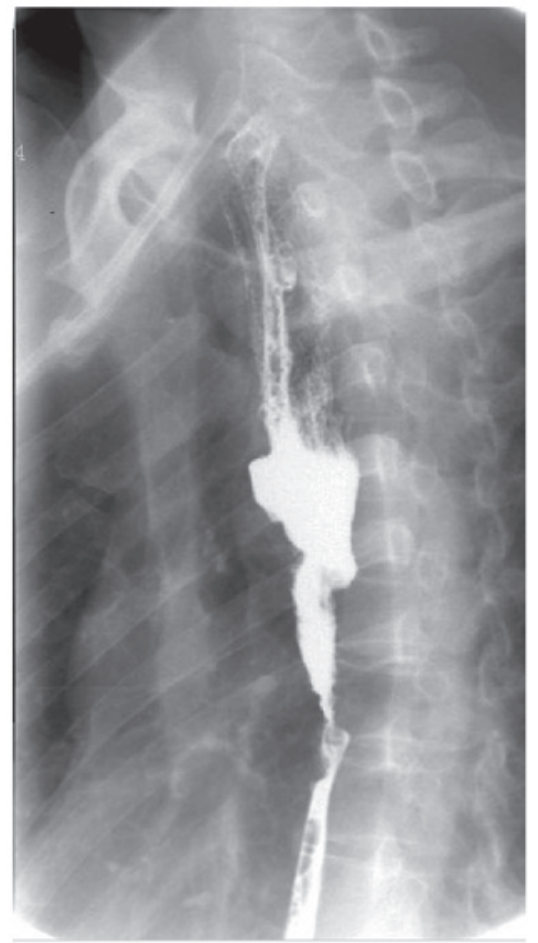

Figure 2. Image from the upper gastrointestinal series (barium swallow) test showing a mucosal lesion, filling defect and wall stiffness along $56 \mathrm{~mm}$ of the esophagus below the arcus aortae. The barium had difficulty in passing through that region, indicating that the proximal esophagus was dilated.

that a mucosal lesion, filling defect and wall stiffness were present $56 \mathrm{~mm}$ along the esophagus, below the level of the arcus aortae. In addition, the barium had difficulty in passing through that region, suggesting that the proximal esophagus was dilated (Fig. 2). Based on the aforementioned findings, mid-esophageal cancer was considered as a possible diagnosis. A contrast-enhanced chest computed tomography scan revealed wall thickening and narrowing of the lumen in the thoracic segment of the esophagus, as well as dilation of the 
upper segment of the esophagus accompanied by the presence of excess fluid. Gastroscopy (CV-260; Olympus Corporation, Tokyo, Japan) revealed nodular irregular ulcers with thick fur on the surface, and surrounding lip-like mucosa $25-30 \mathrm{~cm}$ from the incisor. These lesions were considered to have caused the narrowing of the lumen, which made the advancing of the endoscope difficult (Fig. 3). The patient was suspected to have mid-esophageal cancer and the biopsy performed confirmed the diagnosis of basaloid squamous cell carcinoma (BSC), due to the pathological features observed in the excised tissue; cells possessed round or oval nuclei with 'dusty' chromatin, pale nucleoplasms and small distinct nucleoli, and the tumor lobules had central comedo-type necrosis and peripheral palisading.

Written informed consent was obtained from the patient for the publication of the present study.

\section{Discussion}

Esophageal cancer is the eighth most common type of cancer worldwide, accounting for $2 \%$ of all malignant tumors, and the sixth most common cause of mortality worldwide (1). Furthermore, $75 \%$ of the patients presenting with this type of tumor succumb within the first year of diagnosis, with a 5-year survival rate of $<5 \%$, due to its invasiveness and difficulty in diagnosis (10). Therefore, the early diagnosis of esophageal cancer can markedly improve the survival time of patients.

Esophageal cancer has two pathological subtypes: Squamous carcinoma and adenocarcinoma. BSC comprises a rare subtype of squamous carcinoma found mainly in the upper respiratory and digestive tracts, which was first reported by Wain et al (11) in 1986. A previous study reported the incidence of BSC to be $<1 \%$ (12). This type of carcinoma derives from the fundus of pseudostratified columnar epithelium in the esophageal gland ducts or primitive acinar cells; the mucosa is complete without damage in the early phase, and the tumor grows under the mucosa. Tumor growth oppresses the mucous epithelium, resulting in damage that can cause an ulcer. A previous study of 10 cases reported that BSC patients are typically older males with a history of drinking and smoking (11); however, increasing incidence rates of BSC have been observed in young females and individuals who do not smoke or consume alcohol. In the present case, the patient was a 48-year-old female.

Despite the fact that the patient developed dysphagia 5 months ago, solid and liquid food could be swallowed, although vomiting was occasionally noted afterwards. The symptoms were not aggravated and the results from tests performed at local hospitals did not reveal any presence of cancer 3 months prior to the current admission to the Sir Run Run Shaw Hospital; however, 1 week prior to admission, the patient suddenly presented with regurgitation and difficulty in swallowing even liquids. It is known that the mucosa is not damaged in the early phases of the disease, which may result in a negative upper endoscopic examination, despite the growth of the tumor beneath the mucosa, causing the symptoms to appear only when esophageal motility is affected (13).

HRM is performed as follows: 36 Solid-state channels are spaced at $1-\mathrm{cm}$ intervals, with 12 sensors distributed evenly around each channel, for a total of 432 pressure sensors in a

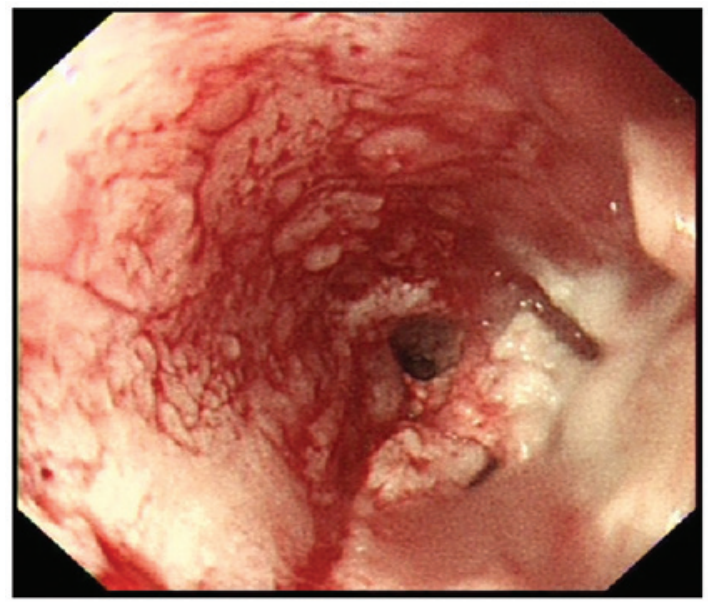

Figure 3. Gastroscopy imaging results revealing the presence of nodular irregular ulcers with thick fur on the surface and surrounding lip-like mucosa $25-30 \mathrm{~cm}$ from the incisor. These lesions resulted in the narrowing of the lumen, which made the advancement of the endoscope difficult.

electrode catheter, with a diameter of $4.7 \mathrm{~mm}$. The technique can therefore simultaneously measure esophageal tension, peristalsis, and the length and pressure of the sphincter. Based on the anatomical features of the esophagus, only two high-pressure zones in the upper esophageal sphincter and LES can be visualized at rest. In the present patient, a high-pressure zone was observed $33 \mathrm{~cm}$ from the incisor, with synchronous waves, indicating achalasia. The patient was $155 \mathrm{~cm}$ tall; the LES in individuals of this height is known to be $40 \mathrm{~cm}$ from the incisor (14). An unexpected low-pressure zone, which relaxed after swallowing $5 \mathrm{ml}$ of water, was observed $6 \mathrm{~cm}$ below the high-pressure zone. It was determined that the low-pressure zone was in the LES, while the high-pressure zone was caused by an obstruction, which was confirmed by an upper gastrointestinal series (barium swallow) test and gastroscopy.

The present results suggested that physicians should focus attention on abnormal high-pressure zones when measuring esophageal pressure. Physiological stricture due to compression by the arcus aortae should be excluded as a cause first. In certain cases, compression by the arcus aortae or an enlarged heart could be responsible, but this could readily be distinguished by counting the number of beats $(3,15,16)$. In addition, physicians should also take into account compression caused by foreign bodies or conditions that can affect the esophagus, such as tight belts, hiatus hernia and esophageal narrowing caused by an ulcer or a tumor. Prior to examining the patient, it is important to remove their belt in order to make sure that any abnormal high-pressure zones observed are not caused by pressure on the gastric area. During data analysis, it is important to determine the pressure inversion point in the gastroesophageal junction, to exclude a large giant hiatus hernia.

A dilated esophagus can induce synchronous waves, thus, organic diseases should be excluded as the cause when patients are diagnosed with achalasia. The presence of synchronous waves, as well as diffuse and segmental esophageal spasms, is commonly observed in patients with achalasia (3).

Overall, it is possible that the presence of cancer was not reported by endoscopy, since the early phases of a sub-mucosal lesion or motility disorder of the esophagus do not result in 
changes that can be detected endoscopically; therefore, for those patients presenting with evident symptoms of esophageal motor dysfunction without significant findings by gastroscopy, HRM is recommended and further examination or follow-ups are required. Finally, esophageal carcinoma should be considered when an abnormal high-pressure zone is observed by HRM.

\section{Acknowledgements}

This study was supported by the Zhejiang Province Key Science and Technology Innovation Team (grant no. 2013TD13) and the Health Department of Zhejiang Province (grant no. 2014KYB121).

\section{References}

1. Parkin DM, Bray F, Ferlay J and Pisani P: Global cancer statistics, 2002. CA Cancer J Clin 55: 74-108, 2005.

2. Devesa SS, Blot WJ and Fraumeni JF Jr: Changing patterns in the incidence of esophageal and gastric carcinoma in the United States. Cancer 83: 2049-2053, 1998.

3. Kruger D: Assessing esophageal dysphagia. JAAPA 27: 23-30, 2014.

4. Fox MR and Bredenoord AJ: Oesophageal high-resolution manometry: Moving from research into clinical practice. Gut 57: 405-423, 2008.

5. Bredenoord AJ and Smout AJ: High-resolution manometry of the esophagus: more than a colorful view on esophageal motility? Expert Rev Gastroenterol Hepatol 1: 61-69, 2007.

6. Pandolfino JE, Fox MR, Bredenoord AJ and Kahrilas PJ: High-resolution manometry in clinical practice: Utilizing pressure topography to classify oesophageal motility abnormalities Neurogastroenterol Motil 21: 796-806, 2009.
7. Bredenoord AJ, Fox M, Kahrilas PJ, Pandolfino JE, Schwizer W and Smout AJ; International High Resolution Manometry Working Group: Chicago classification criteria of esophageal motility disorders defined in high resolution esophageal pressure topography. Neurogastroenterol Motil 24 (Suppl 1): 57-65, 2012.

8. Kahrilas PJ, Ghosh SK and Pandolfino JE: Esophageal motility disorders in terms of pressure topography: The Chicago Classification. J Clin Gastroenterol 42: 627-635, 2008.

9. Bredenoord AJ and Smout AJ: High-resolution manometry. Dig Liver Dis 40: 174-181, 2008.

10. Hiyama T, Yoshihara M, Tanaka S and Chayama K: Genetic polymorphisms and esophageal cancer risk. Int J Cancer 121: 1643-1658, 2007.

11. Wain SL, Kier R, Vollmer RT and Bossen EH: Basaloid-squamous carcinoma of the tongue, hypopharynx, and larynx: Report of 10 cases. Hum Pathol 17: 1158-1166, 1986.

12. Epstein JI, Sears DL, Tucker RS and Eagan JW Jr: Carcinoma of the esophagus with adenoid cystic differentiation. Cancer 53: 1131-1136, 1984.

13. Tsang WY, Chan JK, Lee KC, Leung AK and Fu YT: Basaloid-squamous carcinoma of the upper aerodigestive tract and so-called adenoid cystic carcinoma of the oesophagus: The same tumour type? Histopathology 19: 35-46, 1991

14. Chen J, Qiao RM and Shang J: The relationship between positions of upper and lower esophageal sphincter and height and age. Zhonghua Xiaohua Neijing Zazhi 23: 126-127, 2006 (In Chinese).

15. Kahrilas PJ, Kim HC and Pandolfino JE: Approaches to the diagnosis and grading of hiatal hernia. Best Pract Res Clin Gastroenterol 22: 601-616, 2008.

16. Hou X (ed): Techniques for high resolution manometry. In: High Resolution Manometry in Digestive Tract. Science Press, Beijing, pp158, 2014. 